\title{
Diabetic ketoacidosis presented with COVID-19 infection: A rare case report
}

\author{
Deniz Çekiç ${ }^{1}$, Selçuk Yaylacıํ, Sümeyye Çekiç ${ }^{1}$, Kubilay İşsever ${ }^{1}$, Hamad Dheir ${ }^{1}$, Havva Kocayigit ${ }^{2}$, Mehmet \\ Halil Öztürk ${ }^{3}$, Oğuz Karabay ${ }^{4}$ \\ ${ }^{1}$ Department of Internal Medicine, Faculty of Medicine, Sakarya University, Sakarya, Turkey \\ ${ }^{2}$ Department of Intensive Care, Education and Research Hospital, Sakarya University, Sakarya, Turkey \\ ${ }^{3}$ Department of Radiology, Education and Research Hospital, Sakarya University, Sakarya, Turkey \\ ${ }^{4}$ Department of Infectious Diseases, Faculty of Medicine, Sakarya University, Sakarya, Turkey
}

Received: 2020-11-06.

Accepted: 2020-12-04

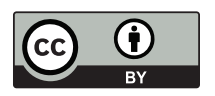

This work is licensed under a

Creative Commons Attribution 4.0 International License

J Clin Med Kaz 2021; 18(1):79-81

Corresponding author:

Deniz Çekiç.

Email: decekic@gmail.com;

ORCID: https://orcid.org/0000-0002-7114-9334

\section{Abstract}

SARS-Corona Virus-2 (SARS-CoV-2/COVID-19) is a corona virus that first appeared in China in December 2019, causing severe acute respiratory failure. The severe infectious disease caused by SARS-Corona Virus- 2 is announced as a pandemic in March 2020 by the WHO. It has infected 4.5 million people and caused the death of 300 thousand people until today (17.05.2020). Although there may be atypical presentations, common complaints are mostly fever, cough, shortness of breath. Increased acute phase reactants commonly spotted as laboratory findings. A peripheral infiltrations in the bilateral lungs and ground glass opacities are characteristic findings in CT as a radiological findings. But some rare presentation also has been reported. In this case, we aimed to present Covid-19 infection presenting with diabetic ketoacidosis in hospital application.

Key words: diabetes mellitus, COVID-19, hyperglycemia

\section{Introduction}

SARS-Corona Virus-2 (SARS-CoV-2/COVID-19) is a corona virus that first appeared in China in December 2019, causing severe acute respiratory failure [1-3]. The severe infectious disease caused by SARS-Corona Virus-2 is announced as a pandemic in March 2020 by the WHO. It has infected 4.425 .485 people and caused the death of 302.059 people until today (17.05.2020). Mortality rate varies between $2-10 \%$ according to the studies conducted [4]. Although there are typical clinical symptoms reported as cough, fever, shortness of breath, there are also serious conditions such as ARDS, multiple organ failure, coma and death [5,6]. Diabetic ketoacidosis is a life-threatening acute complication of diabetes [7]. In this case, we aimed to present Covid-19 infection presenting with diabetic ketoacidosis in hospital application.

\section{Case-presentation}

A 57-year-old male patient with known diabetes and hypertension applied to the emergency room with the complaint of weakness. Glucose: $310 \mathrm{mg} / \mathrm{dl}$, Ph: 7,25, Hco3: $12 \mathrm{mmol} / \mathrm{L}$ TIT keton +++, D-Dimer: 8130 (0-500 $\mu \mathrm{gFEU} / \mathrm{L}), \mathrm{C}-\mathrm{Reactive}$ Protein (CRP): 6,56 (0-5 mg/L),
LDH: 268 (0-248 U/1), Ferritin: 277,84 (21,81-274,66 $\mu \mathrm{g} / \mathrm{L})$, albumin: $3,2(3.5-5.3 \mathrm{mg} / \mathrm{dl}), \mathrm{WBC}$ : 7,43 (4,610,2 K/uL) Neutrophil: 6,22 (2-6,9 K/uL), Lymphocyte: 0,75 (0,6-3,4 K/uL), Platelet: 206000 (142000-424000 $\mathrm{K} / \mathrm{uL}$ ) was spotted as a laboratory findings. Kidney and liver function test was normal. Patient was diagnosed with diabetic ketoacidosis in the light of laboratory values (Table 1) and started on bolus isotonic fluid and insulin infusion treatment. CT scan performed because of dyspnea and image shown compatible viral pneumonia (Thorax CT showed: In the right lung upper lobe anterior segment periphery, the lesion consistent with the halo finding and in the left lobe upper lobe adjacent to the posterior superior section of the arcus aorta was spotted the ground glass opacities (Figure 1A-B). Patient hospitalized with diagnosis of suspicious of COVID-19 and diabetic ketoacidosis. Vital findings at the time of hospitalization fever: $36,4 \mathrm{oC}$, spo2: \%85, blood pressure: $90 / 60 \mathrm{mmHg}$, pulse: $100 / \mathrm{min}$ was spotted. Nasopharyngeal and oropharyngeal Covid PCR were negative twice. The patient, who is the clinical, laboratory and thorax CT compatible with covid infection, was started with $2 \times 200 \mathrm{mg}$ /day plaquenil $2 \times 400 \mathrm{mg} /$ day after loading dose and oseltamivir $2 \times 75 \mathrm{mg}$ /day. On the 


\begin{tabular}{|l|l|l|}
\hline PARAMETER & APPLY & NORMAL \\
\hline Glucose (mg/dl) & 310 & $70-126$ \\
\hline Ure (mg/dl) & 31 & $17-43$ \\
\hline Creatine (mg/dl) & 0.94 & $0,67-1,17$ \\
\hline Alt (U/L) & 16 & $0-50$ \\
\hline Ast (U/L) & 16 & $0-50$ \\
\hline Na (mmol/L) & 134 & $136-146$ \\
\hline K (mmol/L) & 3.8 & $3,5-5,1$ \\
\hline Crp (mg/L) & 6.5 & $0-5$ \\
\hline $\begin{array}{l}\text { Beta-Hydroxibutirat } \\
\text { (mmol/L) }\end{array}$ & 4.5 & $0,5-0,9$ \\
\hline Ph & 7.2 & $7,35-7,45$ \\
\hline Hco3 (mmol/L) & 17 & $22-26$ \\
\hline Pco2 (mmHg) & 43 & $35-48$ \\
\hline Wbc (k/uL) & 7.43 & $4600-10200$ \\
\hline Neu (k/uL) & 6.22 & $2000-6900$ \\
\hline Lymphocyte & 0.753 & \\
\hline Hb (g/dl) & 15.9 & $12,2-18$ \\
\hline Plt (k/uL) & 206000 & $142000-424000$ \\
\hline D Dimer & 8130 & \\
\hline Ldh & 268 & \\
\hline Ferritin & 277 & \\
\hline Hba1c & 10.3 & \\
\hline
\end{tabular}

2nd day of hospitalization, the patient's oral intake allowed when ph: 7.34 Hco3: $23 \mathrm{mmol} / \mathrm{L}$ was detected as urine ketone + . The patient's Hba1C: $10.3 \%$ was detected and basal bolus insulin therapy was performed after blood glucose follow-up was regulated. After completed Covid infection treatment; patient who regulated blood glucose are started basal bolus insulin and patient discharged.

\section{Discussion}

The most common symptoms of admission were fever, cough, sputum and myalgia at Coronavirus infections. Less frequently, headache, shortness of breath, abdominal pain / diarrhea, sore throat and chest pain have been reported $[1-3,5,8]$. Application symptoms of our case, it is seen that it is compatible with the typical symptoms reported. In a meta-analysis, it was reported that white blood cell, neutrophil count, CRP, Ferritin, D-dimer and lactate increased, lymphocyte count, hemoglobin, platelet count and albumin decreased in COVID-19 patients. These findings have also been shown to be associated with mortality $[1-3,8]$. In this case, patient had increased Ferritin, D-dimer, CRP and white blood cell count.
Figure 1 - In the right lung lobe anterior segment periphery, the lesion consistent with the halo finding and in the left lobe upper lobe, the ground glass opacities adjacent to the posterior section of the arcus aorta

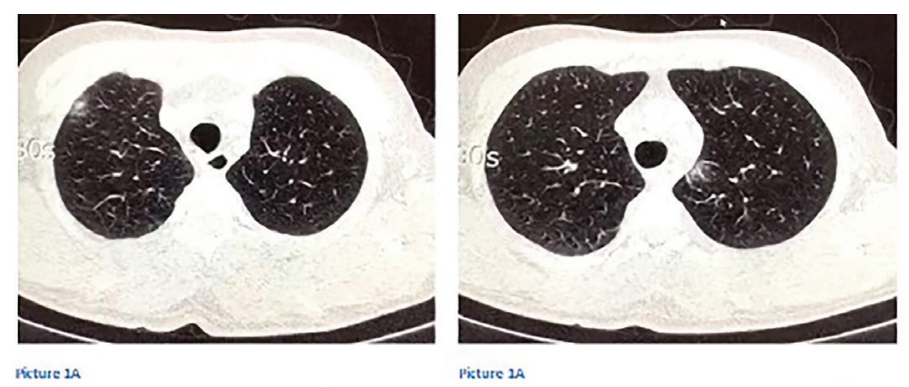

In a different study, diabetes was the second most common comorbid disease in $17 \%$ of 710 COVID-19 cases [9]. In another study with 174 cases, it was stated that diabetes was associated with rapid progression and severe disease progression in the course of Covid-19 [10].

Although standard reference test is seen as PCR in the diagnosis of Covid-19, it is stated that Thorax CT can be used in Covid-19 diagnosis by some sources because of discussions about sensitivity and the fact that PCR test may be false negative/ positive [11].

In the literature, COVID-19 and diabetic ketoacidosis associations have been reported as rare case reports [12]. In the case report of 2 cases related to COVID-19 infection and acute hyperglycemic crisis, a 59-year-old male patient was reported to have cardiac and respiratory mortality on the 16th day of his hospitalization [13].

In this case clinical and laboratory findings were compatible with COVID-19, but the PCR test was negative. After typical Covid-19 findings in thorax CT, the patient was accepted as COVID-19 and treatment was started. With plaquenil treatment and diabetic ketoacidosis treatment, our patient's dyspnea regressed, acidosis improved, blood glucose regulated and patient was discharged and redirected outpatient clinic controls.

\section{Conclusion}

The risk of mortality and morbidity from acute complications of diabetes due to Covid-19 infection is high; and patients should be informed as COVID-19 infection may be more severe in this patient group. During the pandemic periods; diabetic patients, hospital applications should be questioned more carefully and examinations and treatments should be planned quickly.

Disclosures: There is no conflict of interest for all authors.

\section{References}

1. Huang C, Wang Y, Li X, et al. Clinical features of patients infected with 2019 novel coronavirus in Wuhan, China. Lancet. 2020; 395:497-506. doi: 10.1016/S0140-6736(20)30183-5

2. Zhu N, Zhang D, Wang W, et al. A novel coronavirus from patients with pneumonia in China, 2019. N Engl J Med. 2020; 382:727-733. doi: 10.1056/NEJMoa2001017

3. Gorbalenya AE, Baker SC, Baric RS, et al. Severe acute respiratory syndrome-related coronavirus: the species and its viruses - a statement of the coronavirus study group. bioRxiv. 2020; 02.07.937862. doi: https://doi.org/10.1101/2020.02.07.937862.

4. Coronavirus disease (COVID-2019) situation reports 117 . https://www.who.int/docs/default-source/coronaviruse/situationreports/20200516-covid-19-sitrep-117.pdf?sfvrsn=8f562cc_2) (16.05.2020)

5. Chen N, Zhou M, Dong X, et al. Epidemiological and clinical characteristics of 99 cases of 2019 novel coronavirus pneumonia in Wuhan, China: a descriptive study. Lancet. 2020; 395:507-513. doi: 10.1016/S0140-6736(20)30211-7 
6. Chung M, Bernheim A, Mei X, et al. CT imaging features of 2019 novel coronavirus (2019-nCoV). Radiology. 2020; 295:202-207. doi: 10.1148/radiol.2020200230

7. Dhatariya KK, Glaser NS, Codner E, Umpierrez GE. Diabetic ketoacidosis. Nat Rev Dis Primers. 2020; 6(1):40. doi: 10.1038/s41572020-0165-1.

8. Henry BM, de Oliveira MHS, Benoit S, Plebani M, Lippi G. Hematologic, biochemical and immune biomarker abnormalities associated with severe illness and mortality in coronavirus disease 2019 (COVID-19): a meta-analysis. Clin Chem Lab Med. 2020; 58(7):10211028. doi: 10.1515/cclm-2020-0369.

9. Yang X, Yu Y, Xu J, Shu H, Xia J, Liu H, Wu Y, Zhang L, Yu Z, Fang M, Yu T, Wang Y, Pan S, Zou X, Yuan S, Shang Y. Clinical course and outcomes of critically ill patients with SARS-CoV-2 pneumonia in Wuhan, China: a single-centered, retrospective, observational study. Lancet Respir Med. 2020. doi: 10.1016/S2213-2600(20)30079-5

10. Guo W, Li M, Dong Y, Zhou H, Zhang Z, Tian C, Qin R, Wang H, Shen Y, Du K, Zhao L, Fan H, Luo S, Hu D. Diabetes is a risk factor for the progression and prognosis of COVID-19. Diabetes Metab Res Rev. 2020; e3319. doi: 10.1002/dmrr.3319.

11. Gündüz Y, Öztürk MH, Tomak Y. The usual course of Thorax CT findings of Covid-19 infection and When should control Thorax CT scan? Turk J Med Sci. 2020; 50(4):684-686. doi: 10.3906/sag-2004-293

12. Chee YJ, Ng SJH, Yeoh E. Diabetic ketoacidosis precipitated by Covid-19 in a patient with newly diagnosed diabetes mellitus. Diabetes Res Clin Pract. 2020; 164:108166. doi: 10.1016/j.diabres.2020.108166.

13. Kim NY, Ha E, Moon JS, Lee YH, Choi EY. Acute Hyperglycemic Crises with Coronavirus Disease-19: Case Reports. Diabetes Metab J. 2020; 44(2):349-353. doi: 10.4093/dmj.2020.0091. 